\title{
Calculation of the normal and shear compliances of a three-dimensional crack taking into account the contact between the crack surfaces
}

\author{
R. L. Lapin ${ }^{1, \dagger}$, V.A. Kuzkin ${ }^{1,2}$ \\ †lapruslan@gmail.com
}

${ }^{1}$ Peter the Great Saint Petersburg Polytechnic University, 29 Polytechnicheskaya St., St. Petersburg, 195251, Russia

${ }^{2}$ Institute for Problems in Mechanical Engineering RAS, 61 Bolshoj Av. VO, St. Petersburg, 199178, Russia

The calculation of the effective mechanical properties of materials with cracks is a long-standing problem in micromechanics. Usually each crack is modeled by a mathematical cut with traction free surfaces. In this case, the normal and shear compliances of the crack are approximately equal. Then the effective elastic properties of the material with randomly located and arbitrary oriented cracks are orthotropic. If the partial contact between the crack surfaces is taken into account, then the normal and shear compliances are generally different, and the effective properties are no longer orthotropic. This paper proposes a three-dimensional model for estimation of the effect of contacts on the normal and shear compliances of the crack. An infinite flat crack is considered. The surfaces of the crack are connected by a doubly periodic system of identical contacts. Each contact has the shape of a rectangular parallelepiped. The centers of the contacts form a square lattice. The influence of contact sizes and distance between contacts on the ratio of the normal and shear compliances of the crack is investigated. The compliances are calculated numerically using the finite element method. Calculations are carried out for a periodic cell containing a single contact. It is shown that in all considered cases, the normal compliance of the crack is less than shear compliance. The difference between the compliances increases with increasing contact height. A comparison with similar results obtained in a two-dimensional formulation is carried out. It is shown that in the twodimensional case the ratio of compliances is larger than in the three-dimensional case.

Keywords: cracks, contacts, compliance, effective properties, finite element method.

УДК: 531

\section{Вычисление нормальной и сдвиговой податливостей трехмерной трещины с учетом контакта между берегами}

\author{
Лапин Р. Л., ${ }^{1,}$, Кузькин В. А. ${ }^{1,2}$ \\ ${ }^{1}$ Санкт-Петербургский политехнический университет Петра Великого, ул. Политехническая, 29, \\ С.-Петербург, 195251, Россия \\ ${ }^{2}$ Институт проблем машиноведения РАН, Большой пр. ВО, 61, С.-Петербург, 199178, Россия
}

Одна из задач микромеханики состоит в вычислении эффективных механических свойств материалов, содержащих
трещины. Как правило, каждая трещина моделируется математическим разрезом, поверхности которого свободны
от напряжений. При этом нормальная и сдвиговая податливости трещины оказываются равными (в двумерном
случае) или близкими (в трехмерном случае). В результате эффективные упругие свойства материала, содержащего
случайно расположенные и произвольно ориентированные трещины, близки к ортотропным. Учет частичного
контакта между берегами трещин приводит к тому, что нормальная и сдвиговая податливости существенно
отличаются, а эффективные свойства перестают быть ортотропными. В настоящей работе предлагается
трехмерная модель, позволяющая оценить влияние контактов на нормальную и сдвиговую податливости трещины.
Рассматривается бесконечная трещина, берега которой соединены двоякопериодической системой одинаковых
контактов. Каждый контакт имеет форму прямоугольнго параллелепипеда. Центры контактов образуют
квадратную решетку. Исследуется влияние размеров контакта и расстояния между ними на отношение нормальной 
и сдвиговой податливостей трещины. Податливости определяются численно с использованием метода конечных элементов. Расчеты проводятся для ячейки периодичности, содержащей одиночный контакт. Показано, что во всех рассмотренных случая нормальная податливость трещины существенно меньше сдвиговой. При этом чем больше высота контакта по отношению к его ширине, тем сильнее отличаются податливости. Проведено сравнение с известными результатами, полученными в двумерной постановке. Показано, что в двумерном случае отношение нормальной и сдвиговой податливостей больше, чем в трехмерном. Однако, характер зависимости отношения податливостей от расстояния между контактами одинаков.

Ключевые слова: трещины, контакты, податливость, эффективные свойства, метод конечных элементов.

\section{1. Введение}

Одной из актуальных задач микромеханики материалов является определение связи эффективных упругих и прочностных свойств материалов с их внутренней структурой [1]. В частности, большой практический интерес представляет исследование влияния трещин и других неоднородностей на свойства горных пород [1-3]. Данные о количестве, форме и размерах неоднородностей могут быть получены, например, с использованием микротомографии $[2,3]$.

Для оценки влияния трещин на свойства материала может использоваться приближение невзаимодействия $[1,4-6]$. В данном приближении, вклад от каждой трещины в эффективные свойства считается независимо. При этом удается получить простые аналитические формулы, связывающие параметры трещин с эффективными упругими свойствами материала. Данные формулы показывают, в частности, что в приближении невзаимодействия эффективные свойства материала с трещинами близки к ортотропным. Как правило, приближение невзаимодействия применимо в случае, если трещины достаточно удалены друг от друга. В случае близко расположенных трещин (большой плотности трещин) эффективные упругие свойства, как правило, определяются на основе численного решения соответствующих задач теории упругости [7-12]. В частности, в работах [12,13] показано, что упругие свойства материала, содержащего случайно расположенные и произвольно ориентированные трещины, остаются ортотропными при достаточно высоких плотностях трещин. Однако данный результат получен в предположении, что трещина представляет собой идеальный разрез с поверхностями, свободными от напряжений. В реальности трещины имеют шероховатые поверхности, контактирующие между собой, что оказывает существенное влияние на эффективные свойства материала [14-19].

Исследованию влияния контактов между берегами трещин на соотношение их нормальной и сдвиговой податливостей посвящены, например, работы [15-18]. В работе [15] предложен метод вычисления инкрементальной податливости при нормальной нагрузке для трещин с учетом взаимодействия контактов. В работе [16] проведено обобщение данного подхода на случай сдвиговой нагрузки. Обзор работ по вычислению податливости трещин с учетом контактов приведен в работе [17]. Важным ограничением описанных выше работ, является предположение о плоской форме контактов, которое на практике часто не выполняется.
Влияние конечной высоты контактов на упругие свойства горных пород в двумерной постановке исследовалось в работе [19]. Показано, что нормальная и сдвиговая податливости трещины с периодической системой контактов конечной высоты существенно отличаются, в результате чего упругие свойства материала с такими трещинами оказываются неортотропными. В настоящей работе проводится обобщение результатов работы [19] на трехмерный случай.

В данной работе рассматривается бесконечная трещина с двоякопериодической системой одинаковых призматических контактов в трёхмерной постановке. Для вычисления нормальной и сдвиговой податливостей трещины методом конечных элементов решаются две задачи теории упругости для ячейки периодичности, содержащей один контакт. Проводится исследование сходимости по сетке, а также сходимость по размеру расчетной области. Исследуется влияние размеров контактов и расстояния между контактами на отношение нормальной и сдвиговой податливостей. Проводится сравнение с аналогичными результатами, полученными в работе [19] в двумерной постановке.

\section{2. Постановка задачи}

Рассматривается бесконечная плоская трехмерная трещина в линейно-упругой изотропной среде. Берега трещины соединены двоякопериодической системой одинаковых контактов, центры которых образуют двумерную квадратную решетку. Каждый контакт имеет форму прямоугольного параллелепипеда.

В силу периодичности задачи рассматривается ячейка, содержащая один контакт (Рис. 1). Для определения податливостей трещины к верхней и нижней
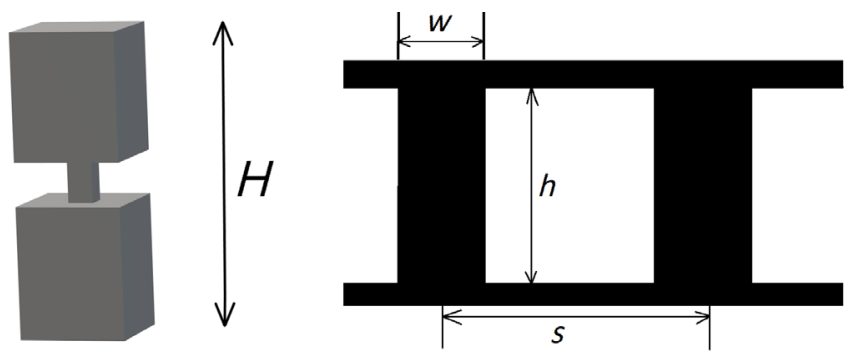

Рис. 1. Геометрические параметры ячейки периодичности: $s-$ расстояние между контактами, $w$ - ширина контакта, $h-$ высота контакта, $H$ - высота ячейки периодичности.

Fig. 1. Geometrical parameters of the periodic cell: $s$ is distance between contacts, $w$ is contact width, $h$ is contact height, $H$ is height of the periodic cell. 
грани ячейки периодичности прикладывается нормальная/сдвиговая равномерно распределенная нагрузка, $\boldsymbol{t}$, единичной интенсивности. В направлениях вдоль трещины ставятся периодические граничные условия. Вычисляется средний вектор скачка перемещений берегов трещины, $[\boldsymbol{u}]$. При известном скачке перемещений и внешней нагрузке нормальная $\left(B_{n}\right)$ и сдвиговая $\left(B_{t}\right)$ податливости вычисляются по формуле:

$$
[\boldsymbol{u}]=\boldsymbol{B} \cdot \boldsymbol{t}, \boldsymbol{B}=B_{n} \boldsymbol{n} \boldsymbol{n}+B_{t}(\boldsymbol{E}-\boldsymbol{n} \boldsymbol{n}) .
$$

Здесь $\boldsymbol{B}$ - тензор податливости трещины; $\boldsymbol{E}$ единичный тензор. Далее исследуется влияние размеров контакта и расстояния между контактами на отношение нормальной и сдвиговой податливостей.

\section{3. Вычисление нормальной и сдви- говой податливостей}

В настоящем параграфе исследуется влияние геометрических параметров контактов и расстояния между ними на отношение нормальной и сдвиговой податливостей трещины.

Для решения поставленной задачи используется метод конечных элементов, реализованный в пакете COMSOL. Данный пакет выбран силу того, что в нем реализованы периодические граничные условия. Для ускорения расчетов строится неравномерная конечно-элементная сетка, показанная на Рис. 2.

При расчетах используются тетраэдрические квадратичные конечные элементы. В результате конечноэлементного расчета вычисляется скачок перемещений берегов трещины, после чего по формуле (1) определяются компоненты тензора податливости.

Для определения необходимого числа конечных элементов проводится исследование сходимости решения по сетке. При исследовании сходимости рассматривается влияние количества элементов, приходящихся на ширину контакта, на величину отношения податливостей $B_{n} / B_{t}$. При этом используются

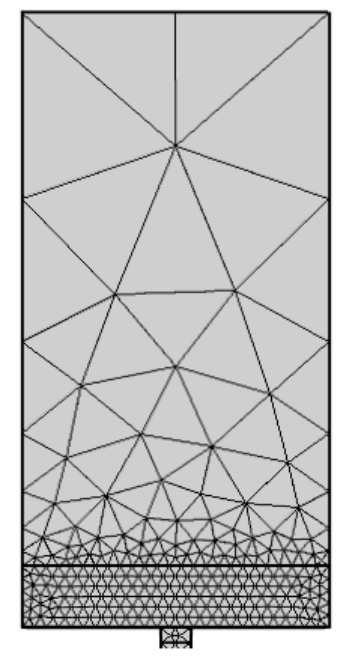

Рис. 2. Пример конечно-элементной сетки. В силу симметрии показана только верхняя часть ячейки периодичности.

Fig. 2. Example of a finite element mesh. For symmetry reasons the upper part of the periodic cell is shown.
Табл. 1. Результаты исследования сходимости по сетке. $N$ число конечных элементов на ширину контакта.

Table 1. Convergence with respect to mesh size. $N$ is a number of finite elements per contact width.

\begin{tabular}{|c|c|}
\hline$N$ & $B_{n} / B_{t}$ \\
\hline 2 & 1.32 \\
\hline 5 & 0.81 \\
\hline 10 & 0.586 \\
\hline 15 & 0.569 \\
\hline 20 & 0.568 \\
\hline 50 & 0.567 \\
\hline
\end{tabular}

следующие значения параметров: $s / w=25, h / w=1$, $H / w=40$. Результаты расчетов с различным числом конечных элементов приведены в Табл. 1. Расчеты показывают, что сходимость практически достигается при использовании 15-ти элементов на ширину контакта. При этом полное число конечных элементов в модели составляет порядка $2 \cdot 10^{4}$.

В численном решении ячейка периодичности имеет конечнуювысоту $H$ (см.Рис. 1). Данный параметр, вообще говоря, влияет на отношение нормальной и сдвиговой податливостей трещины. Для исследования данного влияния определялась зависимость $B_{n} / B_{t}$ от $H / w$ при $s / w=25, h / w=1$. Во всех расчетах использовалось значение коэффициента Пуассона, равное 0.25 . Зависимость отношения нормальной и сдвиговой податливостей от высоты ячейки периодичности представлена на Рис. 3. Видно, что отношение практически сходится к значению, соответствующему бесконечной области при $H / w>20$. В частности, значения $B_{n} / B_{t}$ при $H / w=20$ и $H / w=35$ отличаются примерно на $6 \%$. Далее во всех расчетах использовалось значение $H / w=40$.

В работе [19] в двумерной постановке исследовалось влияние расстояния между контактами на отношение нормальной и сдвиговой податливостей. Сравнение результатов, получающихся в двумерной и трехмерной постановке приведено на Рис. 4. Видно, что в обоих

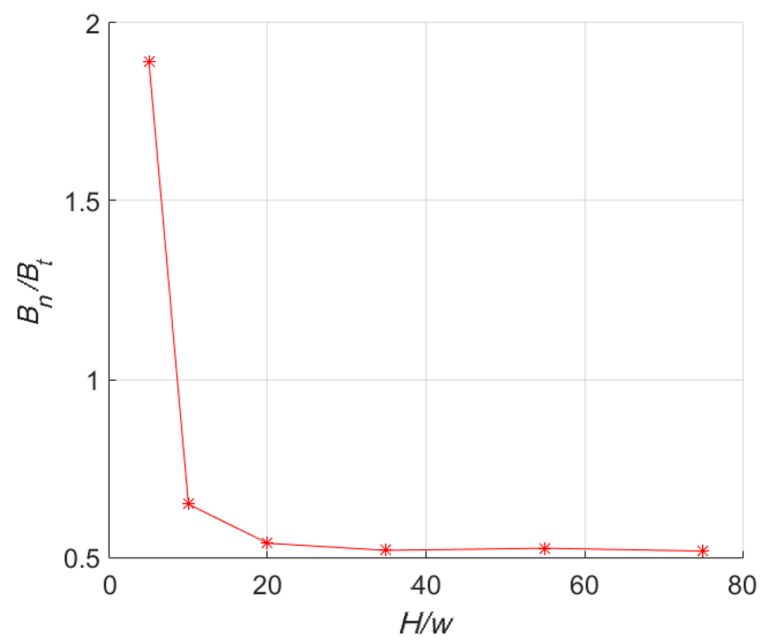

Рис. 3. Зависимость отношения нормальной и сдвиговой податливостей трещины от высоты ячейки периодичности при $s / w=25, h / w=1$.

Fig. 3. Ratio of normal and shear compliances as a function of height of the periodic cell for $s / w=25, h / w=1$. 


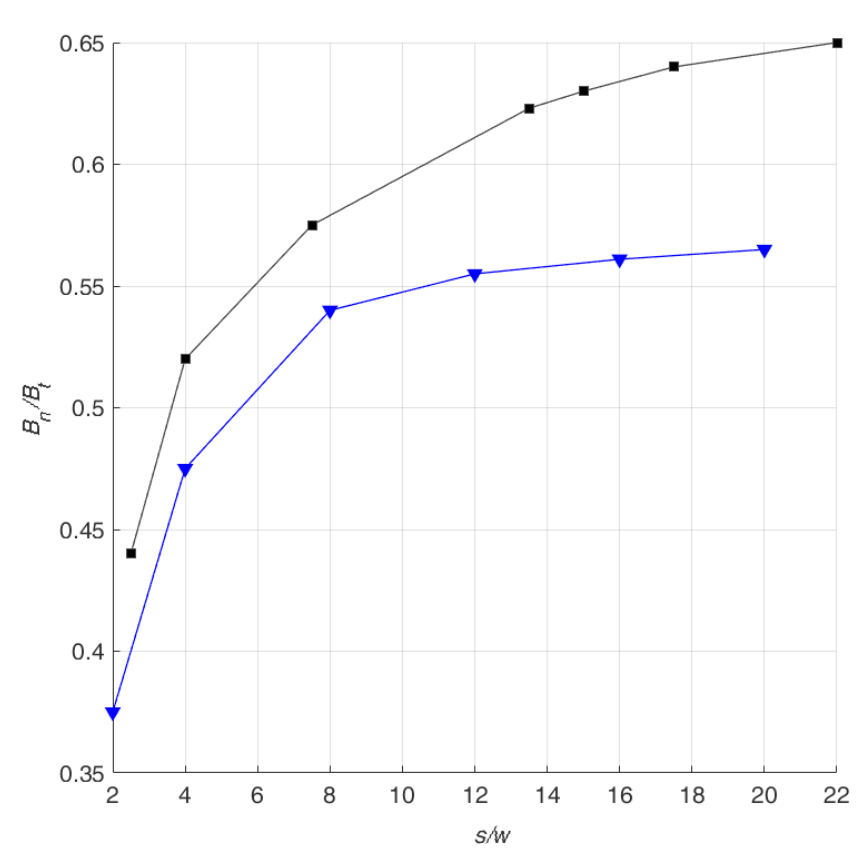

Pис. 4. Зависимость отношений податливостей от расстояния между контактами в двумерной постановке [19] (квадраты) и трехмерной постановке (треугольники) при $h / w=0.44, H / w=40$.

Fig. 4. Ratio of compliances as a function of distance between contacts in $2 \mathrm{D}$ [19] (squares) and 3D (triangles) $(h / w=0.44, H / w=40)$.

случаях увеличение расстояния между контактами приводит к увеличению отношения $B_{n} / B_{t}$. При этом для всех расстояний между контактами значение $B_{n} / B_{t}$ в трехмерной постановке меньше, чем в двумерной.

Для исследования влияния формы контакта (отношения высоты к ширине) на отношение податливостей трещины, проводится серия численных экспериментов при различных значениях $s / w$ и $h / w$. Полученные результаты представлены на Рис. 5. Видно, что при всех рассматриваемых значениях высоты контакта и расстояния между ними нормальная податливость существенно меньше сдвиговой $\left(B_{n} / B_{t}<1\right)$. При увеличении высоты контакта $(h / w)$ различие между податливостями становится больше. Также отметим, что форма контакта сильнее влияет на отношение податливостей, чем расстояние между контактами.

\section{Заключение}

В работе предложена модель трехмерной трещины с двоякопериодической системой одинаковых контактов. Определена зависимость отношения нормальной и сдвиговой податливостей трещины от формы контакта и расстояния между контактами. Показано, что форма контакта (отношение высоты к ширине) влияет на отношение податливостей сильнее, чем расстояние между ними. Во всех рассмотренных случаях нормальная и сдвиговая податливости существенно отличаются. Следовательно, эффективные упругие свойства материала, содержащего трещины с подобными контактами, не ортотропны. Данный факт необходимо учитывать при исследовании упругих свойств горных пород. Полученные результаты имеют важное прикладное значение при анализе механизма разрушения горных пород в процессе их технологической переработки $[3,20]$.

Благодарности/Acknowledgements. Работа выполнена при поддержке Российского научного бонда (грант №17-79-30056). Авторы выражают искреннюю благодарность Л. А. Вайсбергу и М. Л. Качанову за полезные обсуждения./This work was supported by the Russian Science Foundation (Grant No. 17-79-30056). The authors are deeply grateful to L. A. Vaisberg and M. L. Kachanov for stimulating discussions.

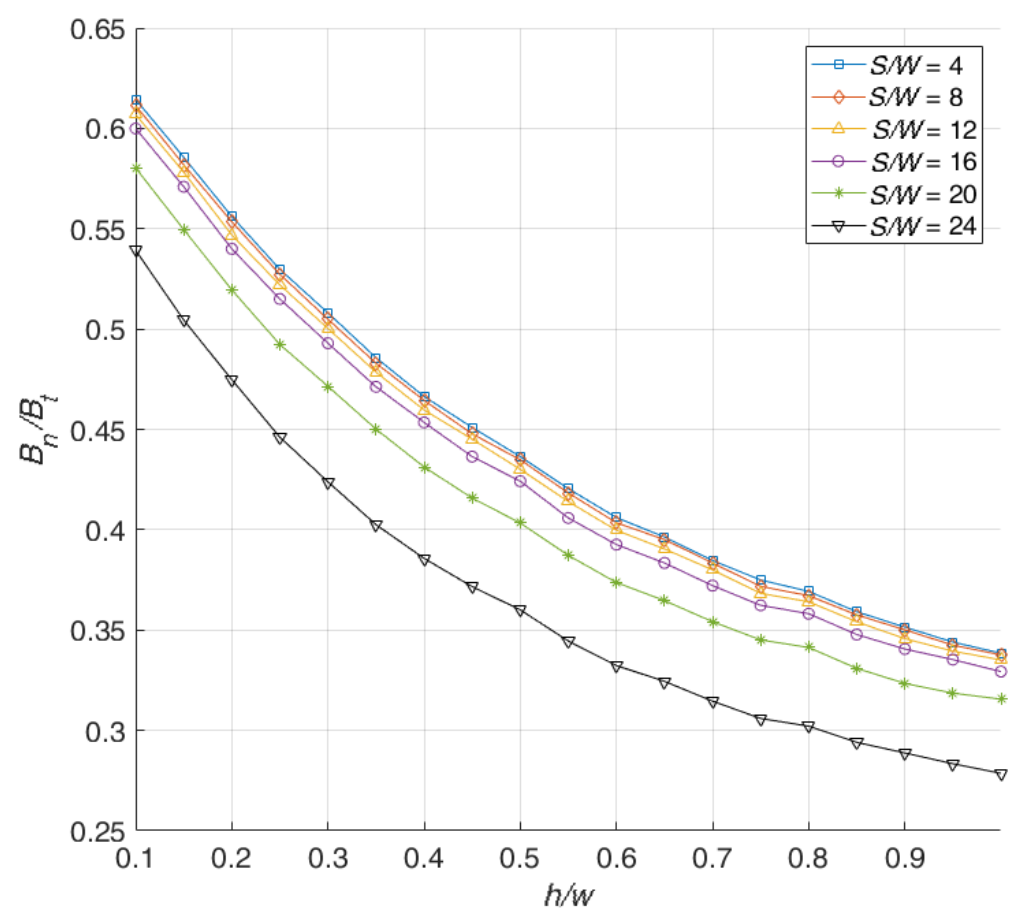

Рис. 5. Зависимость отношения $B_{n} / B_{t}$ от формы контакта $(h / w)$ при различных расстояниях между контактами $(s / w)$.

Fig. 5. Influence of contact shape $(h / w)$ on the ratio of compliances $B_{n} / B_{t}$ for different distances between contacts $(s / w)$. 


\section{Литература/References}

1. M. Kachanov, I. Sevostianov. Micromechanics of materials, with applications. Cham, Springer (2018) 712 p. ․ㅡossref

2. L.A. Vaisberg, E.E. Kameneva, V.S. Nikiforova. Obogashchenie Rud. 3, 51 (2018) (in Russian) [Л.А. Вайсберг, Е.Е. Каменева, В.С. Никифорова. Обогащение руд. 3, 51 (2018).] Crossref

3. L.A. Vaisberg, E.E. Kameneva. Gornyi Zhurnal. 9, 53 (2017). (in Russian) [Л.А. Вайсберг, Е.Е. Каменева. Горный журнал. 9, 53 (2017).] Crossref

4. M. Kachanov. Applied Mechanics Reviews. 45 (8), S304 (1992). Crossref

5. M. Kachanov. Elastic Solids with Many Cracks and Related Problems. In: Advances in Applied Mechanics. Vol. 30 (Ed. by J.W. Hutchinson, T. Wu). New York, Academic Press (1993) p. 256-445 (1993). Crossref

6. V.A. Kuzkin, A.M. Krivtsov, E.A. Podolskaya, M.L. Kachanov. Philosophical Magazine. 96 (15), 1538 (2016). Crossref

7. Z. Hashin. Journal of Mechanics and Physics of Solids. 36, 719 (1988). Crossref

8. Y. Benveniste. Mechanics of Materials. 6, 147 (1987). $\underline{\text { Crossref }}$

9. B. Orlowsky, E. Saenger, Y. Gueguen. International Journal of Fracture. 124, L171 (2003). Crossref

10. A.M. Linkov, S.G. Mogilevskaya, J. A. L. Napiers. International journal of rock mechanics and mining sciences \& geomechanics abstracts. 34 (3-4), 711 (1997). $\underline{\text { Crossref }}$

11. E. Rejwer, L. Rybarska-Rusinek, A. Linkov. Engineering Analysis with Boundary Elements. 43, 105 (2014). Crossref

12. R. L. Lapin, V.A. Kuzkin, M.L. Kachanov. Int. J. Eng. Sci. 124, 16 (2018). Crossref

13. R.L. Lapin, V.A. Kuzkin. Materials Physics and Mechanics. 32 (2), 213 (2017). Crossref

14. S. Laubach. Bulletin of the American Association of Petroleum Geologists. 87, 561 (2003). Crossref

15. J. Greenwood, J. Williamson. Proceedings of the Royal Society of London (A). 295, 300 (1966). Crossref

16. I. Sevostianov, M. Kachanov. Int. J. Eng. Sci. 47, 974 (2009). Crossref

17. M. Ciavarella, G. Demelio, J. Barber, Y. Yang. Proceedings of the Royal Society of London (A). 456, 387 (2000). Crossref

18. V.A. Kuzkin, M.L. Kachanov. International Journal of Engineering Science. 97, 1 (2015). $\underline{\text { Crossref }}$

19. C. Sayers, A. Taleghani, J. Adachi. Geophysical Prospecting. 57, 439 (2009). Crossref

20. V.A. Arsentiev, I.I. Blekhman, L.I. Blekhman, L. A. Vaisberg, K. S. Ivanov, A. M. Krivtsov. Obogashchenie Rud. 1, 30 (2010). (in Russian) [B.А. Арсентьев, И.И. Блехман, Л.И. Блехман, Л.А. Вайсберг, К.С. Иванов, А.М. Кривцов. Обогащение руд. 1, 30 (2010).] 